Article

\title{
Two Approaches to Hamiltonian Bigravity
}

\author{
Vladimir Olegovich Soloviev (iD
}

A. A. Logunov Institute for High Energy Physics, NRC Kurchatov Institute, 142281 Protvino, Russia; vladimir.soloviev@ihep.ru

check for

updates

Citation: Soloviev, V.O. Two

Approaches to Hamiltonian Bigravity.

Universe 2022, 8, 119. https://

doi.org/10.3390/universe8020119

Academic Editors: Chitta Ranjan Das and Alexander S. Barabash

Received: 21 December 2021

Accepted: 7 February 2022

Published: 12 February 2022

Publisher's Note: MDPI stays neutral with regard to jurisdictional claims in published maps and institutional affiliations.

Copyright: (C) 2022 by the author. Licensee MDPI, Basel, Switzerland. This article is an open access article distributed under the terms and conditions of the Creative Commons Attribution (CC BY) license (https:// creativecommons.org/licenses/by/ $4.0 /)$.

\begin{abstract}
Bigravity is one of the most natural modifications of General Relativity (GR), as it is based on the equivalence principle. However, its canonical structure appears rather complicated because of the unusual form of the interaction between two metrics. As a consequence, there are different approaches that are difficult to compare in detail. This work is a first attempt to obtain a synthetic picture of the Hamiltonian formalism for bigravity. Here, we are trying to combine two rather different approaches to gain a binocular view of the theory. The first publications on the subject were based on metric formalism. It was proved that both massive gravity and bigravity with de Rham-Gabadadze-Tolley (dRGT) potential were free of Boulware-Deser (BD) ghosts. This proof was based on the transformation of variables involving a $3 \times 3$-matrix which could be treated as the root of a quadratic equation involving two spatial metrics and a new 3-vector introduced instead of the standard shift variable. Therefore, this matrix occurred as an implicit function of the abovementioned variables. After a substantial amount of time, it became possible to calculate the algebra of constraints in full using this method. However, in another approach also based on metric variables and implicit functions, similar calculations were completed earlier. It is not a new matrix, but the potential itself has been taken as an implicit function of two spatial metrics and four functions constructed of two pairs of lapses and shifts. Finally, a straightforward route to canonical bigravity is to apply tetrad (or vierbein) variables. The matrix square root involved in the dRGT potential can be explicitly extracted if tetrads fulfill the symmetry condition. A full treatment has been developed in first-order formalism by treating tetrads and connections as independent variables. In that case, the theory contains many more variables and constraints than in metric formalism. An essential simplification occurs in second-order vierbein formalism. The potential is given explicitly as a polynomial of bilinear combinations of the two tetrads. The $3 \times 3$-matrix introduced in the pioneer papers can be expressed explicitly through canonical coordinates, and the celebrated transformation of variables arises in the Dirac constraint analysis.
\end{abstract}

Keywords: bimetric gravity; hamiltonian formalism; implicit functions; tetrad approach

\section{Introduction}

Since Fierz-Pauli's work [1], many attempts have been made to construct a nonlinear massive gravity theory. It was necessary to find a non-linear potential as a function of two metric tensors. However, the choice of such a potential is problematic due to the appearance of a 6th degree of freedom [2] called the BD (Boulware-Deser) ghost. After long and sophisticated research, a very special formula has been discovered [3,4]. It is called the dRGT (de Rham-Gabadadze-Tolley) potential. Since this discovery, it has been found that a similar potential had been considered in earlier work [5].

The general idea of bigravity, i.e., a theory of two interacting spin 2 fields, appeared long before the dRGT work. First, the so-called fifth force was discussed in elementary particle physics as a new form of strong interaction [6-9] for spin 2 mesons. Then, a new spin 2 field was treated as a second metric tensor, and the term "bigravity" was born [10].

The great advance achieved in astronomy caused by space exploration has inspired a wide new stream of theoretical research in cosmology and astrophysics. Besides the old difficulty with quantizing gravity, there are now such new challenges as scenarios 
of inflation, the cosmological constant (or dark energy) problem, the dark matter puzzle, etc. A considerable number of physicists have decided that General Relativity needs to be modified to deal with these problems, meaning that revision of GR is now not treated as heresy. Massive gravity and bigravity belong to the vast number of the GR extensions; see, for example, Refs. [11-14] with scalar-tensor theories of gravitation, extra-dimensional theories, theories with Lorentz symmetry violations, string models, and so on.

Recent interest in bigravity has been mainly motivated by the dark energy problem. The theory proposed by Hassan-Rosen $[15,16]$ and based on the dRGT potential admits homogeneous and isotropic time-dependent solutions for two spatial metrics. These cosmological solutions can be interpreted as being driven by a time-dependent dark energy contribution. However, in the study of perturbations around these solutions, exponentially growing instabilities have been found. Now, it is believed that there are no solutions that can replace the standard $\Lambda C D M$ model and predict new physics to be tested by observations.

The non-trivial form of the dRGT potential makes exact calculations difficult. The most convincing non-perturbative proof that Hassan-Rosen bigravity is free of the BD ghost is the analysis of its canonical structure. However, this analysis essentially depends on the choice of variables. Numerous approaches to this problem have been developed, for example, Stückelberg fields, implicit functions [15-21], the tetrad approach, first proposed by Hinterbichler-Rosen [22], and then developed in complex Ashtekar variables [23], in first-order formalism for real variables [24], in second-order tetrad approach [25,26], the geometric mean of two metrics [27], and so on. However, as a rule, the calculations here are so lengthy and involved that every group starts with its own method and notations, and finishes with results formulated in its own way. To be understood by their colleagues, some authors even attach to their work very lengthy special dictionaries for translating notations between different publications; see Refs. [26,27]. We propose consideration of the abovementioned approaches as supplementary to each other, allowing investigation of the same structures from alternative sides.

The purpose of this work is to consider and compare only two approaches. The first is based on metric variables where potential is expressed as an implicit function of metrics or as an explicit function of a new matrix which in turn is an implicit function of the metrics. The second approach is based on tetrad variables. One should be careful when dealing with two space-time metrics as they have two light cones and two definitions of casual structure. Hamiltonian formalism may be constructed only for families of hypersurfaces that are space-like in both metrics. Furthermore, the coordinate lines of time should be time-like in both metrics. These are the necessary conditions to acquire a consistent bigravity theory.

In metric formalism we deal with 20 components of two metrics $g_{\mu v}, f_{\mu v}$, where 12 components are related to spatial 3-metrics $\gamma_{i j}, \eta_{i j}$ induced on hypersurfaces $\tau=$ const, and four lapse and shift functions for each metric $\bar{N}, \bar{N}^{i}, N, N^{i}$. Below, we replace one set of lapse and shift functions with new variables $u$ and $u^{i}$, constructed of lapse and shift ratios; see Equation (11). Then, variations of action over $N$ and $N^{i}$ provide us with the first-class constraints $\mathcal{R}$ and $\mathcal{R}_{i}$, and variations over $u$ and $u^{i}$ will provide second-class constraints $\mathcal{S}$ and $\mathcal{S}_{i}$. This terminology is due to Dirac [28,29]. The induced metrics $\gamma_{i j}$ and $\eta_{i j}$ are the canonical coordinates that are accompanied by the conjugate momenta $\pi^{i j}$ and $\Pi^{i j}$, whereas $u$ and $u^{i}$ play different roles. Variable $u^{i}$ can be excluded by solving constraint equations $\mathcal{S}_{i}=0$, whereas variable $u$ provides a new constraint $\mathcal{S}$. To preserve this constraint in time, one should calculate its Poisson bracket with the Hamiltonian, and this calculation provides a secondary constraint $\Omega$. This pair of constraints occurs as second class as $\{\mathcal{S}, \Omega\} \neq 0$, and excludes the ghost degree of freedom.

Computational difficulties arise because we cannot obtain an explicit formula for potential in terms of the abovementioned variables. Through Poisson bracket calculations, we obtain identities following from the general covariance linear in the potential and its first derivatives. To avoid the ghost degree of freedom, we find that the second derivatives of the potential in $u$ and $u^{i}$ must satisfy the homogeneous Monge-Ampère equation. General solutions of this equation were studied in Fairlie-Leznov's work [30], and their results 
provide us with a method to prove the absence of the BD ghost in a new way. This is an interesting example of the application of implicit function calculus [31] to prove an important physical statement.

The second approach considered in this work allows the development of Hamiltonian formalism of bigravity and to prove the absence of the BD ghost using explicit calculations. It is based on tetrad variables instead of the metric. Indeed, tetrads can be treated as square roots of the metric tensor. Therefore the potential, which is a square root of the two metrics' convolution, can be naturally expressed in tetrad representation. Of course, difficulties arise from the indefinite nature of the space-time metric, and these are discussed in an article by Hassan and Kocic [32]. The probable non-uniqueness and complexity can be avoided under definite conditions formulated there. The symmetry condition for a pair of tetrads is crucial. Such a requirement may seem artificial, but the Hamiltonian approach provides its naturalness. Here, the symmetry condition follows the compatibility of a Hamiltonian constraint with its dynamics. The symmetry conditions in Hamiltonian formalism are divided into a pair of 3-component equations. One equation provides three secondary constraints of the second class, another determines three Lagrange multipliers standing at the primary constraints. Remarkably, this fixing of Lagrange multipliers coincides with the original Hassan-Rosen transformation invented in their first proof of BD ghost absence [17]. Therefore, the use of tetrad variables in Hamiltonian second-order formalism in bigravity gives us numerous advantages:

- potential can be explicitly expressed in canonical coordinates;

- it follows from standard constraint analysis that symmetry conditions for the pair of tetrads is not postulated;

- Hassan-Rosen transformation of variables also follows from constraints treatment according to the Dirac algorithm;

- coefficients in Poisson algebra of the key second-class constraint $\mathcal{S}$ are explicitly expressed through canonical coordinates.

This article is organized as follows. In Section 2, metric formalism and the implicit method are considered. Section 3 is devoted to the tetrad approach. In Section 4, some conclusions are given, and Appendix A contains the expressions of the dRGT potential and constraint $\Omega$.

We prefer to use the same notations as Refs. $[19,20,26]$. In particular, for space-time coordinate indices running from 0 to 3, we use small Greek letters; for internal indices running from 1 to 3 we use small Latin letters from the beginning of the alphabet; for spatial indices, small Latin letters from the middle of the alphabet are used; for internal indices running from 0 to 3 , capital Latin letters are used. Of course, we consider only such couples of metrics that have common time-like and space-like vectors. When the same letter is used for analogous quantities constructed from the first $f_{\mu \nu}$ or with the second $g_{\mu \nu}$ metric, then an upper bar refers to the second one. Some additional notation is explained in Appendix A.

\section{Method of Implicit Functions}

The bigravity Lagrangian includes a sum of the two copies of the GR Lagrangian

$$
\begin{aligned}
& \mathcal{L}^{(f)}=\frac{1}{\kappa^{(f)}} \sqrt{-f} f^{\mu v} R_{\mu \nu}^{(f)}+\mathcal{L}_{M}^{(f)}\left(\psi^{A}, f_{\mu \nu}\right), \\
& \mathcal{L}^{(g)}=\frac{1}{\kappa^{(g)}} \sqrt{-g} g^{\mu v} R_{\mu \nu}^{(g)}+\mathcal{L}_{M}^{(g)}\left(\phi^{A}, g_{\mu \nu}\right),
\end{aligned}
$$

minus the interaction potential

$$
\mathcal{L}=\mathcal{L}^{(f)}+\mathcal{L}^{(g)}-U\left(f_{\mu v}, g_{\mu v}\right),
$$

where $\kappa^{(f)}$ and $\kappa^{(g)}$ are the two gravitational coupling constants, $\mathcal{L}_{M}^{(f)}$ and $\mathcal{L}_{M}^{(g)}$ are Lagrangians of the two species of matter fields symbolically denoted as $\psi^{A}$ and $\phi^{A}$ minimally 
interacting with the corresponding metrics, and $R_{\mu v}^{(f)}, R_{\mu v}^{(g)}$ are Ricci tensors formed of the two metrics. Lagrangian equations are written as systems of two GR equations for the two space-time metrics, where the sources are, correspondingly, two energy-momentum tensors for the two species of matter and the specific tensors algebraically formed of the two metric tensors. Of course, these tensors are equal to the corresponding variational derivatives of the potential. Due to the second Noether theorem, their correspondent covariant derivatives are identically zero.

$$
\begin{aligned}
& R^{(g) \mu v}-\frac{1}{2} g^{\mu v} R^{(g)}=\frac{\kappa^{(g)}}{2}\left(T^{\left(M_{g}\right) \mu v}+T^{(g) \mu v}\right), \quad T^{(g) \mu v}=-\frac{2}{\sqrt{-g}} \frac{\partial U}{\partial g \mu v}, \\
& \bar{\nabla}_{\nu} T^{(g) \mu v} \equiv 0 \\
& R^{(f) \mu v}-\frac{1}{2} g^{\mu \nu} R^{(f)}=\frac{\kappa^{(f)}}{2}\left(T^{\left(M_{f}\right) \mu v}+T^{(f) \mu v}\right), \quad T^{(f) \mu v}=-\frac{2}{\sqrt{-f}} \frac{\partial U}{\partial f_{\mu \nu}}, \\
& \nabla_{v} T^{(f) \mu \nu} \equiv 0 .
\end{aligned}
$$

General covariance demands that $U\left(f_{\mu v}, g_{\mu \nu}\right)$ must be a function of invariants of the tensor $\mathrm{Y}_{v}^{\mu}=g^{\mu \alpha} f_{\alpha \nu}$. However, as a rule, such functions provide theories with a superficial ghost degree of freedom as has been shown in reference [2]. An exception, found in the dRGT work [3], is a potential equal to a linear combination of symmetric polynomials for matrix $\mathrm{X}_{v}^{\mu}=\sqrt{\mathrm{Y}}_{v}^{\mu}$

$$
U=\sqrt{-g} \sum_{i=0}^{i=4} \beta_{i} e_{i}(\mathrm{X})
$$

where $\beta_{i}$ are arbitrary coefficients with dimensionality mass to the fourth degree. At the same time, this construction does not allow the acquisition of an explicit formula for $U$ as a function of canonical coordinates. Even the expression for matrix $Y$ written in components of metrics $g_{\mu v}, f_{\mu \nu}$ looks unappealing. Therefore we proposed use of the Kuchar̆-York covariant approach [33-38] based not on the coordinate but on non-holonomic $3+1$ formed of the unit normal $n^{\alpha}$ to the hypersurface $\tau=$ const and three vectors tangential to the hypersurface $e_{i}^{\alpha}=\partial X^{\alpha} / \partial x^{i}$ and also tangential to internal coordinate lines $X^{\alpha}\left(x^{i}\right)$. The space-time coordinate system $X^{\alpha}$ is arbitrary and does not depend on a choice of the oneparametrical family of space-like hypersurfaces. This choice is specified by four embedding functions $X^{\alpha}=e^{\alpha}\left(\tau, x^{i}\right)$. Arnowitt-Deser-Misner (ADM) formalism [39] is a special case when these embedding functions are chosen as follows:

$$
X^{0}=\tau, \quad X^{i}=x^{i} .
$$

In bigravity, we deal with two space-time metrics $f_{\mu v}, g_{\mu v}$, there are also two bases $\left(n^{\alpha}, e_{i}^{\alpha}\right),\left(\bar{n}^{\alpha}, e_{i}^{\alpha}\right)$, and we are free to choose one of them. These bases differ only in the timelike unit normal $n^{\alpha}$ or $\bar{n}^{\alpha}$ respectively. Let us take $f_{\mu \nu}$ and therefore $n^{\alpha}$ for this purpose, then $f_{\mu \nu}$ is specified by only six components in the decomposition over the space-time basis vectors $\left(n^{\alpha}, e_{i}^{\alpha}\right)$, whereas the second metric $g_{\mu \nu}$ has 10 components when decomposed in this basis

$$
\begin{aligned}
& f^{\alpha \mu}=-n^{\alpha} n^{\mu}+\eta^{i k} e_{i}^{\alpha} e_{k}^{\mu}, \\
& g^{\alpha \mu}=g^{\perp \perp} n^{\alpha} n^{\mu}+g^{\perp k} n^{\alpha} e_{k}^{\mu}+g^{i \perp} e_{i}^{\alpha} n^{\mu}+g^{i k} e_{i}^{\alpha} e_{k}^{\mu} .
\end{aligned}
$$

Let us stress that the lapse and shift functions corresponding to both metrics here are not specified by popular ADM formulae. Lapse and shift $N, N^{i}$ are defined as follows

$$
\dot{e}^{\alpha}=N n^{\alpha}+N^{i} e_{i}^{\alpha}
$$


whereas in the alternative basis they are different

$$
\dot{e}^{\alpha}=\bar{N} \bar{n}^{\alpha}+\bar{N}^{i} e_{i}^{\alpha} .
$$

It is suitable to work with new variables $u$ and $u^{i}$, which are introduced instead of the second pair of lapse and shift $\bar{N}, \bar{N}^{i}$

$$
u=\frac{\bar{N}}{N}, \quad u^{i}=\frac{\bar{N}^{i}-N^{i}}{N}
$$

they can also be expressed as follows:

$$
u=\frac{1}{\sqrt{-g^{\perp \perp}}} \equiv \frac{1}{\sqrt{-g^{\mu v} n_{\mu} n_{v}}}, \quad u^{i}=-\frac{g^{\perp i}}{g^{\perp \perp}} \equiv \frac{g^{\mu v} n_{\mu} e_{\nu}^{i}}{g^{\alpha \beta} n_{\alpha} n_{\beta}},
$$

and they are coefficients relating the two bases

$$
\bar{n}_{\mu}=u n_{\mu}, \quad \bar{e}_{\mu}^{i}=e_{\mu}^{i}-u^{i} n_{\mu}, \quad \bar{n}^{\mu}=\frac{1}{u} n^{\mu}-\frac{u^{i}}{u} e_{i}^{\mu} .
$$

Interestingly, it is possible to develop full Hamiltonian formalism with all constraints and to derive their algebra without an explicit formula for the potential in metric variables. The first construction was proposed in 2011 [15-18]. Then, a different approach was developed by in 2013 [20] (2013), and then in 2018 [21]. In massive gravity, this problem was first solved in 2011 [17] and independently solved by Comelli et al. [40-42] in 2013. In the former works [15-18], the key step was in the change of variables; in our notations it looked like

$$
u^{i}=\left(\delta_{j}^{i}+u D_{j}^{i}\right) v^{j} .
$$

By varying the action over $v^{i}$, the authors have acquired constraint equations, in principle solvable for $v^{i}$.

After the exclusion of these variables, it was necessary to prove: first, that the variational derivative of the action over $u$ provided a new constraint independent of $u$; second, that preservation of this constraint in time required a secondary constraint; and third, that these two constraints had a non-zero Poisson bracket, i.e., they were second class. Then, the exclusion of the BD ghost was proved.

However, what was the meaning of nine variables $D_{j}^{i}$, introduced in the transformation (14)? They should not depend on $u$, and therefore, they should be functions of the spatial metrics $\gamma_{i j}, \eta_{i j}$, and $v^{i}$. They were to be symmetric and satisfy a matrix quadratic equation

$$
D^{i j}=D^{j i}, \quad \gamma^{i j}=D_{k}^{i} v^{k} D_{m}^{j} v^{m}+\varepsilon^{-2} D^{i k} D_{k}^{j},
$$

where $\varepsilon=\left(1-\eta_{i j} v^{i} v^{j}\right)^{-\frac{1}{2}}$.

Therefore, we again must deal with a matrix square root, in this case for $3 \times 3$-matrix. In work by another group $[19,20]$ Hassan-Rosen transformation (14) was not used, as was also the case in the work by Comelli et al [40-42]. It was proposed that potential is a scalar density depending on two 3-metrics induced on the hypersurfaces and two sets of lapse and shift functions. Let us introduce a new notation $\tilde{U}=U / N$. Then, the bigravity Hamiltonian can be written as follows

$$
\mathrm{H}=\int d^{3} x\left(N \mathcal{H}+N^{i} \mathcal{H}_{i}+\bar{N} \overline{\mathcal{H}}+\bar{N}^{i} \overline{\mathcal{H}}_{i}+N \tilde{U}\right),
$$

where the first and second couple of terms corresponds to the metrics $f_{\mu \nu}$ and $g_{\mu \nu}$, respectively. Below, it is suitable to use variables $u, u^{i}$ introduced in Equation (11) instead of 
$\bar{N}, \bar{N}^{i}$. As the theory is generally covariant, the Hamiltonian should contain four first-class constraints that generate arbitrary deformations of space-like hypersurfaces and arbitrary transformations of spatial coordinates on them. These constraints can be obtained by varying $\mathrm{H}$ over $N, N^{i}$, and the second-class constraints are derived by varying over $u, u^{i}$

$$
\begin{aligned}
\mathcal{R} \equiv \mathcal{H}+u \overline{\mathcal{H}}+u^{i} \overline{\mathcal{H}}_{i}+\tilde{U}=0, \\
\mathcal{R}_{i} \equiv \mathcal{H}_{i}+\overline{\mathcal{H}}_{i}=0, \\
\mathcal{S} \equiv \overline{\mathcal{H}}+\frac{\partial \tilde{U}}{\partial u}=0, \\
\mathcal{S}_{i} \equiv \overline{\mathcal{H}}_{i}+\frac{\partial \tilde{U}}{\partial u^{i}}=0 .
\end{aligned}
$$

As proved by Teitelboim $[43,44]$ the first-class constraints (17) and (18) should satisfy the following algebra (when all second-class constraints are fulfilled)

$$
\begin{array}{r}
\{\mathcal{R}(x), \mathcal{R}(y)\}=\left[\eta^{i k}(x) \mathcal{R}_{k}(x)+\eta^{i k}(y) \mathcal{R}_{k}(y)\right] \delta, i(x, y), \\
\left\{\mathcal{R}_{i}(x), \mathcal{R}_{k}(y)\right\}=\mathcal{R}_{i}(y) \delta_{, k}(x, y)+\mathcal{R}_{k}(x) \delta_{, i}(x, y), \\
\left\{\mathcal{R}_{i}(x), \mathcal{R}(y)\right\}=\mathcal{R}(x) \delta_{, i}(x, y) .
\end{array}
$$

It was shown [19] that this requirement led to 12 identities for the potential and its first derivatives

$$
\begin{array}{r}
2 \eta_{j k} \frac{\partial \tilde{U}}{\partial \eta_{i j}}+2 \gamma_{j k} \frac{\partial \tilde{U}}{\partial \gamma_{i j}}-u^{i} \frac{\partial \tilde{U}}{\partial u^{k}}=\delta_{k}^{i} \tilde{U}, \\
2 u^{j} \gamma_{j k} \frac{\partial \tilde{U}}{\partial \gamma_{k \ell}}-u^{\ell} u \frac{\partial \tilde{U}}{\partial u}+\left(\eta^{k \ell}-u^{2} \gamma^{k \ell}-u^{k} u^{\ell}\right) \frac{\partial \tilde{U}}{\partial u^{k}}=0 .
\end{array}
$$

Since a bigravity with the general form of scalar potential has the BD ghost [2], one needs an additional condition to be put on the potential.

It was claimed [17] that secondary constraints (19) and (20) should not be solvable for a lapse and shift both in massive gravity and bigravity with the dRGT potential. In our approach, instead of lapse $\bar{N}$ and shift $\bar{N}^{i}$, we use variables $u$ and $u^{i}$, so this claim now looks as follows:

$$
\frac{D\left(\mathcal{S}, \mathcal{S}_{i}\right)}{D\left(u, u^{k}\right)}=0 .
$$

It is evident from the constraint structure (19) and (20) that this Jacobian is really a Hessian

$$
\frac{D\left(\mathcal{S}, \mathcal{S}_{i}\right)}{D\left(u, u^{k}\right)}=\operatorname{det} \mathbf{L}, \quad \text { where } \quad \mathbf{L}=\left\|\frac{\partial^{2} \tilde{U}}{\partial u^{A} \partial u^{B}}(x)\right\| .
$$

Therefore, the potential must satisfy the homogeneous Monge-Ampère equation in non-dynamical variables $u^{A}=\left(u, u^{i}\right)$

$$
\operatorname{det} \mathbf{L}=\operatorname{det}\left\|\frac{\partial^{2} \tilde{U}}{\partial u^{A} \partial u^{B}}(x)\right\|=0 .
$$

The solutions of this equation have been analyzed in a general case in an article by Fairlie and Leznov [30]. The first application of their method to the case of massive gravity appeared in references [40-42]. In bigravity, this method was used in reference [20]. Tensor $\mathrm{Y}_{v}^{\mu}=g^{\mu \alpha} f_{\alpha \nu}$ has the following components in the tensor product of vector bases $\left(n^{\mu}, e_{i}^{\mu}\right) \otimes\left(n_{v}, e_{v j}\right)$ 


$$
\begin{array}{ll}
\mathrm{Y}_{\perp}^{\perp}=-u^{-2}, & \mathrm{Y}_{i}^{\perp}=u^{-2} u_{i}, \\
\mathrm{Y}_{\perp}^{k}=u^{-2} u^{k}, & \mathrm{Y}_{i}^{k}=\gamma^{k j} \eta_{j i}-u^{-2} u^{k} u_{i}
\end{array}
$$

or it can be written in the matrix form as follows:

$$
\mathrm{Y}=\left(\begin{array}{cc}
-u^{-2}\left[n^{\mu} n_{v}\right] & u^{-2} u^{i}\left[n^{\mu} e_{v i}\right] \\
u^{-2} u^{j}\left[e_{j}^{\mu} n_{v}\right] & \left(\gamma^{i j}-u^{-2} u^{i} u^{j}\right)\left[e_{i}^{\mu} e_{v j}\right]
\end{array}\right)
$$

where square brackets are used only to separate the tensor basis from its components.

Next, we need to find necessary and sufficient conditions for the potential to exclude the ghost degree of freedom. It is shown in reference [20] that this condition is the fulfillment of the homogeneous Monge-Ampère equation concerning to variables $u$ and $u^{i}$. It guarantees the functional dependence of the four second-class constraint equations derived as variational derivatives of the action in variables $u$ and $u^{i}$. All the terms of the Hamiltonian are linear in $N$ and $N^{i}$ including the potential term, as it is a scalar density and proportional to $N$. However, only kinetic terms are linear in $u$ and $u^{i}$, and the potential is non-linear when expressed in metric variables. This is a reason that second-class constraints are in the form (19) and (20). Nevertheless, the potential becomes linear in variable $u$ when variable $u^{i}$ is excluded

$$
\tilde{U}=W^{\prime}+u V^{\prime}
$$

If $u^{i}$ is not excluded, we have

$$
W=\tilde{U}-u V-u^{i} V_{i} \equiv U-u \frac{\partial \tilde{U}}{\partial u}-u^{i} \frac{\partial \tilde{U}}{\partial u^{i}} .
$$

The explicit form of the functions $\tilde{U}, V, V^{i}$ and $W$ in canonical variables can be obtained only in tetrad formulation, which is considered in the next section. An exception appears only in the two-dimensional space-time case. The exclusion of variables $u^{i}$ and the Dirac bracket construction are provided in the general form [20]. Equation (20) can be solved for $u^{i}$, and these solutions are substituted in Equation (19) and in the Hamiltonian. Hassan and Rosen were able to express the matrix square root $X_{v}^{\mu}=\sqrt{Y}_{v}^{\mu}$ in new variables $v^{i}, D_{j}^{i}$, introduced by their transformation (14), where $3 \times 3$-matrix $D_{j}^{i}$ was a solution of the quadratic matrix Equation (15), i.e., a matrix square root. In a later work [21], the calculations of all Poisson brackets regarding $D_{j}^{i}$ as an implicit function of the canonical coordinates were made in detail.

In the approach given in reference [20], we first treated $u$ and $u^{i}$ as dynamical variables, and determined their conjugate momenta. These momenta were zero, so they provided primary constraints $\pi_{u^{i}}=0$ which had non-zero Poisson brackets with corresponding secondary constraints Equation (20) Therefore, these six constraints could be excluded by introducing Dirac brackets or by explicitly solving Equation (20) for $u^{i}$. Besides canonical variables for the matter fields, Hamiltonian formalism of bigravity contained 13 pairs of gravitational coordinates and conjugate momenta. However, one of these pairs $\left(u, \pi_{u}\right)$ could be excluded later. The corresponding second-class constraint $\mathcal{S}=0$ appeared on the r.h.s. of the first-class constraint algebra responsible for the general coordinate invariance of the formalism 


$$
\begin{aligned}
\{\mathcal{R}(x), \mathcal{R}(y)\} & =\left(\eta^{i k} \mathcal{R}_{k}+u u^{i} \mathcal{S}\right)(x) \delta, i(x, y) \\
& -\left(\eta^{i k} \mathcal{R}_{k}+u u^{i} \mathcal{S}\right)(y) \delta_{, i}(y, x), \\
\left\{\mathcal{R}_{i}(x), \mathcal{R}(y)\right\} & =\mathcal{R}(x) \delta_{i}(x, y)+u, i \mathcal{S} \delta(x, y), \\
\left\{\mathcal{R}_{i}(x), \mathcal{R}_{j}(y)\right\} & =\mathcal{R}_{j}(x) \delta_{, i}(x, y)-\mathcal{R}_{i}(y) \delta_{, j}(y, x) .
\end{aligned}
$$

The most important results are the Poisson brackets of this constraint

$$
\begin{aligned}
\{\mathcal{S}(x), \mathcal{S}(y)\} & =\left(\Theta^{i}-\bar{U}^{i} \mathcal{S}\right)(x) \delta_{, i}(x, y)-\left(\Theta^{i}-\bar{U}^{i} \mathcal{S}\right)(y) \delta_{, i}(y, x), \\
\{\mathcal{R}(x), \mathcal{S}(y)\} & =\left(u^{i}-u \bar{U}^{i}\right) \mathcal{S}(x) \delta_{, i}(x, y)-\left(u\left(\bar{U}^{i} \mathcal{S}\right)_{, i}+\Omega\right) \delta(x, y), \\
\left\{\mathcal{R}(x), \pi_{u}(y)\right\} & =\mathcal{S}(x) \delta(x, y), \\
\left\{\Omega(x), \pi_{u}(y)\right\} & =\Theta^{i}(x) \delta_{, i}(x, y)-\Theta^{i}(y) \delta_{, i}(y, x), \\
\{\mathcal{S}(x), \Omega(y)\} & \neq 0 .
\end{aligned}
$$

In the first pair of these Equations (37) and (40), there appears a function $\Theta^{i}$, and analysis of the Monge-Ampère equation shows that $\Theta^{i}=0$ in solutions. In Equations (37) and (38) there appears a new function

$$
\bar{U}^{i}=\left\|\frac{\partial^{2} \tilde{U}}{\partial u^{i} \partial u^{j}}\right\|^{-1} \times\left\|\frac{\partial^{2} \tilde{U}}{\partial u \partial u^{j}}\right\|,
$$

for the existence of $\bar{U}^{i}$, it is necessary that matrix $\mathbf{L}$ introduced in (27) be non-degenerate, i.e., it is necessary to have

$$
\operatorname{rank}(\mathbf{L})=3
$$

Equation (38) includes a new function of the canonical variables $\Omega$. To preserve the constraint $\mathcal{S}=0$ in time, we need a condition $\Omega=0$. This is the desired secondary constraint, as it is independent of $u$, according to Equation (39). In the metric approach, we can conclude that $\Omega$ contains only zero- and first-order terms in canonical momenta, and commutes with all the constraints besides $\mathcal{S}$. Therefore, the last non-dynamical variable $u$ is fixed using the following equation

$$
\{\Omega(x), \mathrm{H}\}=\int d^{3} y N(y) u(y)\{\Omega(x), \mathcal{S}(y)\}=0,
$$

and $(\mathcal{S}, \Omega)$ form a pair of second-order constraints required for the exclusion of the BD ghost.

The approach based on implicit functions allows the discovery of necessary and sufficient conditions for the potential constructed as a function of bigravity canonical coordinates required to avoid the BD ghost. These conditions are given by Equations (24), (25), (27) and (43).

The difference between the approaches of references $[17,20,21]$ may be described as follows:

- different implicit functions are used; in the first case it is matrix $D_{j}^{i}$, and in the second case it is the potential $\tilde{U}=U / N$;

- special transformation (14) is required in the first case;

- $\quad$ potential can be displayed almost explicitly, i.e., employing the implicit function $D_{j}^{i}$, in the first case [21]. 


\section{Method of Tetrads}

\subsection{Tetrads in GR}

The drawbacks of an approach based on implicit functions are evident, and it is possible to avoid them by choosing other canonical coordinates. Instead of the induced metrics $\left(\gamma_{i j}, \eta_{i j}\right)$, one can take the spatial components of vierbeins (tetrads). In GR, the fields $E_{\mu}^{A}\left(X^{\alpha}\right)$ of orthonormalized non-coordinate bases

$$
E_{\mu}^{A} E_{v}^{B} h_{A B}=g_{\mu \nu}, \quad E_{\mu}^{A} E_{B}^{\mu}=\delta_{A}^{B}, \quad E_{\mu}^{A} E_{A}^{v}=\delta_{\mu}^{v}
$$

are necessary to use when dealing with fermionic fields of matter, and, of course, they can be used in other cases. The number of field components becomes greater, but the number of transformations conserving Lagrangian invariance also increases. For the Hamiltonian approach, this means that the number of first-class constraints increases. If we have four such constraints in metric formalism, then, in the tetrad approach, the other 6 constraints should be added, providing boosts and spatial rotations

$$
\bar{L}_{A B}=E_{i A} \pi_{B}^{i}-E_{i B} \pi_{A}^{i}=0 .
$$

The space-time metric tensor has 10 independent components, where four of them are serving to construct Lagrange multipliers for first-class constraints in ADM formulation. In the Kuchař-York approach, these Lagrange multipliers also depend on the embedding variables $e^{\mu}\left(\tau, x^{i}\right)$.

The tetrad basis $E_{\mu}^{A}$ has 16 components, four of which can be excluded by taking the unit normal to the hypersurface as the time-like leg of the tetrad $E_{\mu}^{0}=\bar{n}_{\mu}$. Then, we stay with 12 variables $E_{\mu}^{a}$. However, as all of them are now tangential to the hypersurface $\tau=$ const, we can treat them as triads $\mathbf{e}_{i}^{a}=E_{\mu}^{a} e_{i}^{\mu}$ with nine components only. Therefore, if in the metric approach we have six canonical coordinates $\gamma_{i j}$ and four first-class constraints $\mathcal{H} \approx 0, \mathcal{H}_{i} \approx 0$, then in the tetrad formulation we have nine triad components $\mathbf{e}_{i a}$ and should add three additional first-class constraints

$$
\bar{L}_{a b}=\mathbf{e}_{i a} \pi_{b}^{i}-\mathbf{e}_{i b} \pi_{a}^{i}
$$

Of course, the number of degrees of freedom has not changed $6-4=9-7=2$. Please note that if we are free from the condition $E_{\mu}^{0}=n_{v}$, then we need three more constraints.

\subsection{Choice of Tetrad Parametrization in Bigravity}

In the theory of bigravity, we deal with a couple of tetrad bases, but potential is invariant only under diagonal transformations $E_{A}^{\mu}$ and $F_{A}^{\mu}$ both in internal Minkowski space

$$
E_{A}^{\mu} \rightarrow \Lambda_{A}^{B} E_{B^{\prime}}^{\mu}, \quad F_{A}^{\mu} \rightarrow \Lambda_{A}^{B} F_{B^{\prime}}^{\mu}
$$

and in space-time. Therefore, the number of first-class constraints for a tetrad not fixed by the unit normal will be 10 :

$$
\mathcal{H} \approx 0, \quad \mathcal{H}_{i} \approx 0, \quad L_{A B}=F_{i A} \Pi_{B}^{i}-F_{i B} \Pi_{A}^{i} \approx 0
$$

We start with two tetrads $E_{\mu}^{A}$ and $F_{\mu}^{A}$ forming orthonormal bases related to the two space-time metrics. However, we can fix a time-like vector as a unit normal to the hypersurface of state only for one of the two tetrads. Let us take $E_{\mu}^{A}$ in this case and adopt equation $E_{\mu}^{0}=\bar{n}_{\mu}$ for it. To avoid massive calculations, it seems useful in constructing tetrad Hamiltonian formalism to export some results from the metric one following earlier 
works [45-48]. Then, the corresponding metric coordinates and momenta can be expressed using triad variables

$$
\begin{aligned}
\gamma_{i j} & =\mathbf{e}_{i}^{a} \mathbf{e}_{j}^{b} \delta_{a b}, \quad \gamma^{i j}=\mathbf{e}_{a}^{i} \mathbf{e}_{a,}^{j}, \quad \mathbf{e}_{a}^{i} \mathbf{e}_{i b}=\delta_{a b}, \quad \mathbf{e}_{a}^{i} \mathbf{e}_{j a}=\delta_{j}^{i} . \\
\pi^{i j} & =\frac{1}{4}\left(\mathbf{e}^{i a} \pi^{j a}+\mathbf{e}^{j a} \pi^{i a}\right) .
\end{aligned}
$$

The time-like vector in the second tetrad $F_{\mu}^{0}$ has, in general, three non-zero projections on the spatial hypersurface, and these three numbers may serve as its additional coordinates. Let us parametrize these three additional degrees of freedom in another way, i.e., by a parameter of Lorentz transformation that moves a special form of tetrad to the general one ${ }^{1}$

$$
\Lambda_{B}^{A}=\left(\begin{array}{cc}
\varepsilon & p_{b} \\
p^{a} & \mathcal{P}_{b}^{a}
\end{array}\right), \quad \varepsilon=\sqrt{1+p_{a} p^{a}}, \quad \mathcal{P}_{b}^{a}=\delta_{b}^{a}+\frac{1}{\varepsilon+1} p^{a} p_{b}
$$

Then, the canonical coordinates representing the two tetrad bases of the space-time are identified with two triad bases in the hypersurface, i.e., $3 \times 3 \times 2=18$ numbers plus 3 numbers that can be treated as a boost parameter and can transform the second tetrad time-like vector into the corresponding unit normal. The following operator $\mathcal{P}_{b}^{a}$, defined by formula (52) and its inverse, will be widely used below

$$
\mathcal{P}^{-1}{ }_{b}^{a}=\delta_{b}^{a}-\frac{1}{\varepsilon(\varepsilon+1)} p^{a} p_{b}
$$

Triads $\mathbf{f}_{a i}$ are introduced in parallel to triads $\mathbf{e}_{a i}$

$$
\eta_{i j}=\mathbf{f}_{i}^{a} \mathbf{f}_{j}^{b} \delta_{a b}, \quad \eta^{i j}=\mathbf{f}_{a}^{i} \mathbf{f}_{a,}^{j}, \quad \mathbf{f}_{a}^{i} \mathbf{f}_{i b}=\delta_{a b}, \quad \mathbf{f}_{a}^{i} \mathbf{f}_{j a}=\delta_{j}^{i}
$$

It is suitable to replace canonical variables with non-canonical ones with the following notations:

$$
v_{a}=p_{a} / \varepsilon \quad \tilde{f}^{i a}=(\mathcal{P})_{a b}^{-1} \mathbf{f}^{i b},
$$

then

$$
\begin{aligned}
& F_{i}^{a}=\tilde{f}_{a i} \equiv \mathcal{P}_{a b} \mathbf{f}_{b i}, \\
& F_{i}^{0}=\tilde{v}_{i} \equiv v_{a} \tilde{f}_{a i} \equiv p_{a} \mathbf{f}_{a i} \equiv p_{i},
\end{aligned}
$$

and the Poisson brackets are the following:

$$
\begin{aligned}
& \left\{v_{a}(x), \Pi_{0}^{i}(y)\right\}=\tilde{f}^{i a} \delta(x, y), \quad\left\{v_{a}(x), \Pi_{b}^{i}(y)\right\}=-\tilde{f}^{i a} v_{b} \delta(x, y), \\
& \left\{\mathbf{f}_{a i}(x), \Pi_{b}^{j}(y)\right\}=\left(\delta_{i}^{j} \delta_{a b}+\frac{p_{i} p_{b} \mathbf{f}^{j a}}{\varepsilon+1}\right) \delta(x, y), \\
& \left\{\mathbf{f}_{a i}(x), \Pi_{0}^{j}(y)\right\}=-\frac{p_{a} \delta_{i}^{j}+\varepsilon \mathbf{f}^{\mathbf{j} a} p_{i}}{\varepsilon+1} \delta(x, y),
\end{aligned}
$$

Then the variables of the metric formalism are expressed as follows:

$$
\begin{aligned}
\eta_{i j} & =F_{i}^{A} F_{A j}=-\tilde{v}_{i} \tilde{v}_{j}+\tilde{f}_{a i} \tilde{f}_{a j} \equiv-p_{i} p_{j}+\tilde{f}_{a i} \tilde{f}_{a j}, \\
\Pi^{i j} & =\frac{1}{4}\left(p^{i} \Pi_{0}^{j}+p^{j} \Pi_{0}^{i}+\mathcal{P}_{a b}\left(\mathbf{f}^{i a} \Pi^{j b}+\mathbf{f}^{j a} \Pi^{i a}\right)\right) .
\end{aligned}
$$


Poisson brackets are not always identical in metric and tetrad formalisms. They can differ by some expressions proportional to the constraints of tetrad formalism. For example, we can see this for the Poisson bracket of momenta

$$
\begin{aligned}
& \left\{\Pi^{i j}(x), \Pi^{k \ell}(y)\right\}=\frac{1}{4}\left(\eta^{i k} \mathcal{M}^{j \ell}+\eta^{i \ell} \mathcal{M}^{j k}+\eta^{j k} \mathcal{M}^{i \ell}+\eta^{j \ell} \mathcal{M}^{i k}\right), \\
& \left\{\pi^{i j}(x), \pi^{k \ell}(y)\right\}=\frac{1}{4}\left(\gamma^{i k} \overline{\mathcal{M}}^{j \ell}+\gamma^{i \ell} \overline{\mathcal{M}}^{j k}+\gamma^{j k} \overline{\mathcal{M}}^{i \ell}+\gamma^{j \ell} \overline{\mathcal{M}}^{i k}\right) .
\end{aligned}
$$

where

$$
\begin{aligned}
\mathcal{M}^{i j} & =\frac{1}{4}\left(p^{i} \Pi_{0}^{j}-p^{j} \Pi_{0}^{i}+\mathcal{P}_{a b}\left(\mathbf{f}^{i a} \Pi^{j b}-\mathbf{f}^{j a} \Pi^{i b}\right)\right), \\
\mathcal{M}^{i j} & =\frac{1}{4}\left(\mathbf{e}^{i a} \pi_{a}^{j}-\mathbf{e}^{j a} \pi_{a}^{i}\right) .
\end{aligned}
$$

New terms also appear in Poisson brackets of metric Hamiltonian constraints. Something can be improved if we change the definitions of constraints

$$
\begin{aligned}
& \mathcal{H}_{i}=\mathcal{H}_{i M}+\Pi_{0}^{k} p_{k, i}+\Pi_{a}^{k} \tilde{f}_{a k, i}-\left(\Pi_{0}^{k} p_{i}\right)_{, k}-\left(\Pi_{a}^{k} \tilde{f}_{a i}\right)_{, k} \\
& \overline{\mathcal{H}}_{i}=\overline{\mathcal{H}}_{i M}+\pi_{a}^{k} \mathbf{e}_{a k, i}-\left(\pi_{a}^{k} \mathbf{e}_{a i}\right)_{, k} .
\end{aligned}
$$

Then, we obtain

$$
\begin{aligned}
\{\mathcal{H}(x), \mathcal{H}(y)\} & =\eta^{i k}(x) \mathcal{H}_{i}(x) \delta_{, k}(x, y)-\eta^{i k}(y) \mathcal{H}_{i}(y) \delta_{, k}(y, x), \\
\left\{\mathcal{H}_{k}(x), \mathcal{H}(y)\right\} & =\mathcal{H}(x) \delta_{, k}(x, y), \\
\left\{\mathcal{H}_{i}(x), \mathcal{H}_{j}(y)\right\} & =\mathcal{H}_{j}(x) \delta_{, i}(x, y)-\mathcal{H}_{i}(y) \delta_{, j}(y, x), \\
\left\{\mathcal{H}_{k}(x), L_{A B}(y)\right\} & =L_{A B}(x) \delta_{, k}(x, y),
\end{aligned}
$$

and

$$
\begin{aligned}
\{\overline{\mathcal{H}}(x), \overline{\mathcal{H}}(y)\} & =\gamma^{i k}(x) \overline{\mathcal{H}}_{i}(x) \delta_{, k}(x, y)-\gamma^{i k}(y) \overline{\mathcal{H}}_{i}(y) \delta_{, k}(y, x), \\
\left\{\overline{\mathcal{H}}_{k}(x), \overline{\mathcal{H}}(y)\right\} & =\overline{\mathcal{H}}(x) \delta_{, k}(x, y), \\
\left\{\overline{\mathcal{H}}_{i}(x), \overline{\mathcal{H}}_{j}(y)\right\} & =\overline{\mathcal{H}}_{j}(x) \delta_{, i}(x, y)-\overline{\mathcal{H}}_{i}(y) \delta_{, j}(y, x), \\
\left\{\overline{\mathcal{H}}_{k}(x), \bar{L}_{a b}(y)\right\} & =\bar{L}_{a b}(x) \delta_{, k}(x, y) .
\end{aligned}
$$

The main advantage of the tetrad approach is the opportunity to perform the square root of the convolution matrix constructed from the two space-time metric tensors [22]. In a general form, this can be done as follows:

$$
\begin{array}{cc}
\mathrm{g}^{-1} & =\mathrm{E}^{-1}\left(\mathrm{E}^{-1}\right)^{T}, \quad g^{\mu \nu}=E_{A}^{\mu} \mathrm{E}^{A v}, \\
\mathrm{f} & =\mathrm{F}^{T} \mathrm{~F}, \\
\mathrm{X} & =\sqrt{\mathrm{g}^{-1 \mathrm{f}}}=\sqrt{\mathrm{E}^{-1}\left(\mathrm{E}^{-1}\right)^{T} \mathrm{~F}^{T} \mathrm{~F}}=F_{\mu A} F_{\nu}^{A},
\end{array}
$$

if the symmetry condition is valid

$$
\left(\mathrm{FE}^{-1}\right)^{T}=\mathrm{FE}^{-1}
$$

The meaning of (80) lies in providing the equivalence of metric and tetrad expressions for the dRGT potential. The first tetrad formulation was developed in reference [22] where the potential was initially given in the form of a wedge product. It was shown that such a form of potential was equivalent to the dRGT metric formula only if the symmetry 
condition for tetrads were valid. With the given formulae for tetrads, we can calculate matrix $X=\sqrt{Y}$ and obtain the following:

$$
\mathbf{X}_{v}^{\mu}=\left(\begin{array}{cc}
-\frac{\varepsilon}{u}\left[n^{\mu} n_{v}\right] & \frac{p_{a} \mathbf{f}^{j a}}{u}\left[n^{\mu} e_{v j}\right] \\
\frac{\varepsilon u^{i}}{u}-p_{a} \mathbf{e}^{i a}\left[e_{i}^{\mu} n_{v}\right] & -\frac{u^{i} p^{a} \mathbf{f}^{a a}}{u}+\mathbf{f}^{j a} \mathcal{P}_{a b} \mathbf{e}^{i b}\left[e_{i}^{\mu} e_{v j}\right]
\end{array}\right),
$$

The matrix obtained is a mixed $(1,1)$-tensor, the components of which are expressed employing auxiliary variables $u, u^{i}$, the triad components $\mathbf{e}_{i a}, \mathbf{f}_{i a}$ and three components of the boost parameter $p_{a}$. Now it is easy to obtain symmetric polynomials of this matrix, and the general form of dRGT potential. In the tetrad approach, the potential is linear in non-dynamical variables $u$ and $u^{i}$; these expressions are given in detail in Appendix A.

Then, we substitute our parametrization for the different components of tetrads into Equation (80). The conditions of tetrad symmetry in the chosen variables appear as the following equations for the 10 components of the matrix Equation (80):

$$
\begin{gathered}
p_{a}+u p_{b} \mathbf{f}_{b j} \mathbf{e}^{j a}-u^{j} \mathcal{P}_{a b} \mathbf{f}_{b j}=0, \\
\mathbf{f}_{c i} \mathcal{P}_{c[a} \mathbf{e}_{b]}^{i}=0 .
\end{gathered}
$$

The second equation may be written as the symmetry condition of the two triads convolution

$$
z_{a b}-z_{b a}=0, \quad \text { where } \quad z_{a b}=\tilde{f}_{i a} \mathbf{e}^{i b},
$$

and the first one can be solved for $u^{i}$ if we apply the second

$$
u^{i}=v^{i}+u \bar{v}^{i},
$$

where $v_{a}=p_{a} / \varepsilon, v^{i}=v_{a} \mathbf{f}^{i a}, \bar{v}^{i}=v_{a} \mathbf{e}^{i a 2}$. The last equation is equivalent to Hassan-Rosen transformation, and matrix $D_{j}^{i}$ is found to be

$$
D^{i j}=\varepsilon z^{i j} \equiv \varepsilon \tilde{f}^{i a} \mathbf{e}^{j a} .
$$

Then, Equation (15) is satisfied as

$$
D^{i}{ }_{j} v^{j}=\bar{v}^{j}, \quad \varepsilon^{-2} D^{i k} D_{k}^{j}=\mathbf{e}^{i a} \mathcal{P}_{a b}^{-1} \mathbf{f}^{k b} \eta_{k \ell} \mathcal{P}_{c d}^{-1} \mathbf{f}^{\ell d} \mathbf{e}^{j d}=\mathbf{e}^{i a} \mathbf{e}^{j a}-\bar{v}^{i} \bar{v}^{j} .
$$

Similar formulae were obtained in reference [50] (see Equations (3.10) and (3.11) there), but without application to Hamiltonian formalism.

Given these results, matrix $X$ takes the following form

$$
X_{v}^{\mu}=\left(\begin{array}{cc}
-\frac{\varepsilon}{u}\left[n^{\mu} n_{v}\right] & \frac{\varepsilon v^{j}}{u}\left[n^{\mu} e_{v j}\right] \\
\frac{\varepsilon v^{i}}{u}\left[e_{i}^{\mu} n_{v}\right] & \left(z^{i j}-\frac{\varepsilon v_{v}^{i} j}{u}\right)\left[e_{i}^{\mu} e_{v j}\right]
\end{array}\right),
$$

This expression should be compared with the expression for matrix $Y$ given by Equation (31). The most important result states that this square root matrix should depend on $u^{-1}$ instead of $u^{-2}$ dependence of matrix $\mathrm{Y}$. The symmetric polynomials also occur as linear in $u^{-1}$, and, after multiplication on $u$ the potential function, $\tilde{U}$ becomes linear in $u$.

\subsection{The Constraints of Tetrad Bigravity}

If we ignore potential for some time, then, for the gravitational field given by metric $f_{\mu v}$ and expressed in coordinates $\tilde{f}^{i a}, v_{a}$ and momenta $\Pi_{i a}, \Pi_{j 0}$, we will have 10 constraints

$$
L_{a 0}=0, \quad L_{a b}=0, \quad \mathcal{H}=0, \quad \mathcal{H}_{i}=0 .
$$


For another field given by metric $g_{\mu v}$, there are 7 constraint equations

$$
\bar{L}_{a b}=0, \quad \overline{\mathcal{H}}=0, \quad \overline{\mathcal{H}}_{i}=0,
$$

and all these 17 constraints are first class, i.e., their Poisson brackets are zero on constraint solutions.

Given the potential, bigravity action is invariant only under diagonal rotations of the tetrads and diagonal space-time diffeomorphisms. After adding the potential, we see that the theory is invariant only under diagonal transformations, both for coordinates and for internal Minkowski vectors. Therefore, only seven constraints are first class:

$$
\begin{aligned}
\mathcal{R} & \equiv \mathcal{H}+u \overline{\mathcal{H}}+u^{i} \overline{\mathcal{H}}_{i}+\tilde{U} \approx 0, \\
\mathcal{R}_{i} & \equiv \mathcal{H}_{i}+\overline{\mathcal{H}}_{i} \approx 0, \\
L_{a b}^{+} & \equiv L_{a b}+\bar{L}_{a b} \approx 0 .
\end{aligned}
$$

Lagrange multipliers at these constraints are in turn $N, N^{i}, \lambda_{a b}^{+}$. Besides the abovementioned seven, there are another 10 primary constraints. It will be shown in the next subsection that they are second class

$$
\begin{aligned}
\mathcal{S} & \equiv \overline{\mathcal{H}}+\frac{\partial \tilde{U}}{\partial u}=0, \\
\mathcal{S}_{i} & \equiv \overline{\mathcal{H}}_{i}+\frac{\partial \tilde{U}}{\partial u^{i}}=0, \\
L_{a b}^{-} & \equiv L_{a b}-\bar{L}_{a b}=0, \\
L_{a 0} & \equiv \tilde{f}_{i a} \Pi_{0}^{i}+\tilde{v}_{i} \Pi_{a}^{i} .
\end{aligned}
$$

These constraints appear when the action is varied with respect to $u, u^{i}, \lambda_{a b}^{-}, \lambda_{a}$. Let us mention that, given Equations (94) and (95), Equation (91) may be written as follows:

$$
\mathcal{R} \equiv \mathcal{R}^{\prime \prime}+u \mathcal{S}+u^{i} \mathcal{S}_{i} \approx 0, \quad \mathcal{R}^{\prime \prime} \equiv \mathcal{H}+W \approx 0, \quad W \equiv \tilde{U}-u \frac{\partial \tilde{U}}{\partial u}-u^{i} \frac{\partial \tilde{U}}{\partial u^{i}} .
$$

The Hamiltonian is formed as a linear combination of all primary constraints

$$
\mathrm{H}=\int d^{3} x\left(N\left(\mathcal{R}^{\prime \prime}+u \mathcal{S}+u^{i} \mathcal{S}_{i}\right)+N^{i} \mathcal{R}_{i}+\lambda_{a b}^{+} L_{a b}^{+}+\lambda_{a b}^{-} L_{a b}^{-}+\lambda^{a} L_{a 0}\right) .
$$

In the next subsection, we give the results of calculations for Poisson brackets between constraints. It allows the discovery of secondary constraints, to classify constraints into first and second classes, and to find important coefficients in the constraint algebra.

\subsection{The Algebra of Constraints}

The requirement to preserve the primary constraints in evolution leads to the following equations

$$
\begin{aligned}
\dot{\mathcal{S}} & =\{S(x), \mathrm{H}\}=N(x) \Omega(x)+\left(\left(N^{i}+N v^{i}\right)(x) \mathcal{S}(x)\right),{ }_{i} \\
\dot{L}_{a b}^{-} & =\left\{L_{a b}^{-}, \mathrm{H}\right\} \approx 2 \beta_{1} N e u\left(z_{b a}-z_{a b}\right) \equiv 2 \beta_{1} N e u G_{b d}, \\
\dot{L}_{a 0} & =\left\{L_{a 0}, \mathrm{H}\right\} \approx \beta_{1} N e\left(u^{i} \tilde{f}_{i a}-\varepsilon\left(v_{a}+u v_{j} e^{j a}\right)\right) .
\end{aligned}
$$

Equation (100) proves th existence of the secondary constraint that is necessary to exclude the BD ghost $\Omega$; the explicit formula for it is given in Appendix A. Equation (101) are equivalent to half of the tetrad symmetry conditions (82); they are secondary constraints $G_{b d}$ in Hamiltonian formalism. Equation (102) are equivalent to Equation (85) and determine Lagrange multipliers $u^{i}$; they also coincide with Hassan-Rosen transformation (14). 
The following equations confirm that constraints $L_{a b}^{-}, G_{c d}, L_{a 0}, \mathcal{S}_{i}$ are second class

$$
\begin{aligned}
\left\{L_{a b}^{-}(x), G_{c d}(y)\right\} & =\left[\delta_{a c} z_{(b d)}-\delta_{a d} z_{(c b)}-\delta_{b c} z_{(a d)}+\delta_{b d} z_{(c a)}\right] \delta(x, y) \neq 0, \\
\left\{L_{a 0}(x), \mathcal{S}_{i}(y)\right\} & =e \tilde{f}_{b i}\left[\beta_{1} \delta_{b a} e_{0}(z)+\beta_{2}\left(\delta_{b a} e_{1}(z)-z_{b a}\right)\right. \\
& \left.+\beta_{3}\left(\delta_{b a} e_{2}(z)+z_{b c} z_{c a}-z z_{b a}\right)\right] \delta(x, y) \neq 0 .
\end{aligned}
$$

All the constraints are invariant under spatial coordinate transformations, and so they commute with the generator $\mathcal{R}_{k}$. The first-class constraints $\mathcal{R}, \mathcal{R}_{k}$ commute with each other (34)-(36) if the second-class constraint $\mathcal{S}=0$ is satisfied. The most interesting are the following relationships:

$$
\begin{aligned}
& \{\mathcal{S}(x), \mathcal{S}(y)\}=\bar{v}^{i} \mathcal{S}(x) \delta_{, i}(x, y)-\bar{v}^{i} \mathcal{S}(y) \delta_{, i}(y, x) \\
& \{\mathcal{R}(x), \mathcal{S}(y)\}=\left(u^{i}+u \bar{v}^{i}\right) \mathcal{S}(x) \delta_{, i}(x, y)+\left(u\left(\bar{v}^{i} \mathcal{S}\right)_{, i}-\Omega\right) \delta(x, y) \\
& \{\mathcal{S}(x), \Omega(y)\} \neq 0 .
\end{aligned}
$$

They guarantee the exclusion of the BD ghost. Non-dynamical variable $u$ is determined from a condition of the second-class constraint $\Omega$ preservation in time

$$
\dot{\Omega}=0 \approx \int d^{3} x N\{\Omega, \mathcal{R}\} \approx \int d^{3} x N\left(\left\{\Omega, \mathcal{R}^{\prime}\right\}+u\{\Omega, \mathcal{S}\}\right)=0,
$$

where $\mathcal{R}^{\prime}=\mathcal{R}-u \mathcal{S}$. If we compare coefficients in Poisson algebra derived with tetrads (105) and (106) to the coefficients discovered from the implicit function method (37) and (38), we discover that the following relationships are valid

$$
\bar{U}^{i}=\left\|\frac{\partial^{2} \tilde{U}}{\partial u^{i} \partial u^{j}}\right\|^{-1} \times\left\|\frac{\partial^{2} \tilde{U}}{\partial u \partial u^{j}}\right\|=-\bar{v}^{i} \equiv-\mathbf{e}^{i a} \mathbf{v}_{a} .
$$

\section{Conclusions}

This work considers and compares two alternative methods of constructing canonical formalism of bigravity theory for the first time. One of them is based on metric variables and their implicit functions. Another method exploits tetrads and is formulated explicitly. We believe that these two approaches are the simplest compared to others, and that a synthetic view of them provides a better understanding of bigravity theory.

In the first works on the Hamiltonian structure of bigravity, the absence of the BD ghost was confirmed, and the self-consistency of bigravity was proved using metric formalism. The advantage of this approach is in the minimal set of variables involved and as a consequence of a minimal number of second-class constraints derived. There is only one pair of these constraints required to remove the Boulware-Deser ghost. However, the disadvantage is in involving implicit functions and the necessity of blind calculations. It is important to mention that there are two different methods belonging to the metric approach. In the first one [15-18,21], the crucial role is played by the remarkable Hassan-Rosen transformation of variables, which has been a deep insight into the structure of the dRGT potential. The second method [20] does not use any guesses, and is based on more or less standard calculations with Poisson brackets plus the Fairlie-Leznov results [30] on the Monge-Ampère equation.

The second method for treating the problem considered in this work is based on tetrad formalism. Its advantage lies in the opportunity to obtain an explicit expression for dRGT potential and to avoid any implicit functions in the calculations. This approach allows the derivation of all the constraint structure of the theory, including the most important pair of second-class constraints, removing the Boulware-Deser ghost. The symmetry condition for tetrads providing equivalence to the metric approach, first considered in Hinterbichler-Rosen [22], directly follows from standard Dirac constraint analysis. Additionally, the Hassan-Rosen transformation of variables [17] that has been crucial for the first pa- 
pers automatically follows from the tetrad Hamiltonian formalism in a form free from any implicit functions. Of course, this approach involves more canonical variables, and contains additional constraints, both of the first and second class. This may be considered a disadvantage, but is not a big one.

The unified picture of these two routes to canonical bigravity given in this work does not yet include the first-order tetrad formalism developed in articles [23,24] and some other work. Nevertheless, we hope that moving in the direction of combining and uniting the original approaches proposed by different groups of researchers will allow the painting of to a simple geometrical picture useful for applying to physical reality.

Attempts to apply the theory of bigravity to generate new cosmological scenarios up to now have not been very successful, due to the appearance of instabilities or to results indistinguishable from $\Lambda \mathrm{CDM}$ model predictions. However, not all opportunities have been studied yet. The discovery of a new successful cosmological model may inspire a new deep study of canonical formalism, bearing in mind, for example, the Cauchy problem (see reference [51]), perturbation theory, numerical methods, canonical quantization, etc.

Funding: This research received no external funding.

Conflicts of Interest: The author declares no conflict of interest.

\section{Appendix A}

In this Appendix, we collected the most cumbersome formulae. They provide the explicit expression of the dRGT potential defined by Equation (5) and the secondary constraint $\Omega$ arising from Equation (106).

The first expression for the potential appears when we substitute matrix $X$ given by Equation (81) into Equation (5)

$$
\begin{aligned}
U & =N\left(u V+u^{i} V_{i}+W\right), \\
V & =e\left(\beta_{0} e_{0}(z)+\beta_{1} e_{1}(z)+\beta_{2} e_{2}(z)+\beta_{3} e_{3}(z)\right), \\
V_{i} & =-\mathbf{f}_{i a} C_{a b} p_{b} \\
W & =e\left(\beta_{0} e_{0}(w)+\beta_{1} e_{1}(w)+\beta_{2} e_{2}(w)+\beta_{3} e_{3}(w)\right),
\end{aligned}
$$

where $e=\operatorname{det}\left(\mathbf{e}_{a i}\right), f=\operatorname{det}\left(\mathbf{f}_{a i}\right), u=\frac{\bar{N}}{N}, u^{i}=\frac{\bar{N}^{i}-N^{i}}{N}, e_{i}$ are symmetric polynomials of $(3 \times 3)$-matrices $z_{a b}, w_{a b}, x_{a b}$, defined as follows

$$
\begin{aligned}
z_{a b} & =\mathcal{P}_{a c} x_{c b} \equiv \tilde{f}_{i a} \mathbf{e}^{i b}, \quad \mathcal{P}_{a c}=\delta_{a c}+\frac{p_{a} p_{c}}{\varepsilon+1}, \\
w_{a b} & =\mathcal{P}_{a c}^{-1} x_{c b} \equiv \tilde{f}^{i a} \eta_{i j} \mathbf{e}^{j b}, \quad \mathcal{P}_{a c}^{-1}=\delta_{a c}-\frac{p_{a} p_{c}}{\varepsilon(\varepsilon+1)}, \\
x_{c b} & =\mathbf{f}_{i c} \mathbf{e}^{i b}, \quad \tilde{f}_{i a}=\mathcal{P}_{a c} \mathbf{f}_{i c}, \quad \tilde{f}^{i a}=\mathcal{P}_{a c}^{-1} \mathbf{f}^{i c}, \\
C_{a b} & =e\left[\beta_{1} \delta_{b a} e_{0}(x)+\beta_{2}\left(\delta_{b a} e_{1}(x)-x_{b a}\right)\right. \\
& \left.+\beta_{3}\left(\delta_{b a} e_{2}(x)+x_{b c} x_{c a}-x x_{b a}\right)\right] .
\end{aligned}
$$

After substitution $u^{i}=v^{i}+u \bar{v}^{i}$ i.e., the decrypted Hassan-Rosen transformation we obtain a simplified result:

$$
\tilde{U} \equiv \frac{U}{N}=u V^{\prime}+W^{\prime}
$$

where

$$
\begin{aligned}
V^{\prime} & =e\left(\beta_{1} e_{1}(w)+\beta_{2} e_{2}(w)+\beta_{3} e_{3}(w)\right)+\beta_{0} e \\
W^{\prime} & =\frac{e}{\varepsilon}\left(\beta_{1} e_{0}(z)+\beta_{2} e_{1}(z)+\beta_{3} e_{2}(z)\right)+\beta_{4} f .
\end{aligned}
$$


The secondary constraint $\Omega$ has the following appearance

$$
\begin{aligned}
\Omega & =\frac{\partial \mathcal{H}}{\partial \Pi_{a}^{i}} \frac{\partial V^{\prime}}{\partial \tilde{f}_{a i}}-\frac{\partial \overline{\mathcal{H}}}{\partial \pi_{a}^{i}} \frac{\partial W^{\prime}}{\partial e_{a i}} \\
& -v^{i} \tilde{f}_{a k, i} \frac{\partial V^{\prime}}{\partial \tilde{f}_{a k}}-\bar{v}^{i} e_{a k, i} \frac{\partial W^{\prime}}{\partial e_{a k}} \\
& +v_{, k}^{i}\left(e_{a i} \frac{\partial}{\partial e_{a k}}-\delta_{i}^{k}\right) V^{\prime}+\bar{v}^{i}\left(e_{a i} \frac{\partial W^{\prime}}{\partial e_{a k}}\right)_{, k} .
\end{aligned}
$$

\section{Notes}

Below we will see that $\varepsilon$ introduced in (52) coincides with the notation $\varepsilon$ introduced in Equation (15).

It was shown for the first time in reference [49].

\section{References}

1. Fierz, M.; Pauli, W. On relativistic wave equations for particles of arbitrary spin in an electromagnetic field. Proc. R. Soc. Lond. 1939, A173, 211-232.

2. Boulware, D.G.; Deser, S. Can gravitation have a finite range? Phys. Rev. 1972, D6, 3368-3382. [CrossRef]

3. De Rham, C.; Gabadadze, G.; Tolley, A.J. Resummation of Massive Gravity. Phys. Rev. Lett. 2011, 106, 231101. [CrossRef] [PubMed]

4. De Rham, C.; Gabadadze, G.; Tolley, A.J. Ghost free Massive Gravity in the Stückelberg language. Phys. Lett. 2012, B711, 190-195. [CrossRef]

5. Zumino, B. Effective Lagrangians and broken symmetries. In Brandeis Univ. Lectures on Elementary Particles and Quantum Field Theory; MIT Press: Cambridge, MA, USA, 1970; Volume 2, p. 437.

6. Salam, A.; Strathdee, J. Nonlinear Realizations. I. The Role of Goldstone Bosons. Phys. Rev. 1969, 184, 1750-1759. [CrossRef]

7. Salam, A.; Strathdee, J. Nonlinear Realizations. II. Conformal Symmetry. Phys. Rev. 1969, 184, 1760-1768. [CrossRef]

8. Isham, C.J.; Salam, A.; Strathdee, J. Spontaneous breakdown of conformal symmetry. Phys. Lett. 1970, B31, 300-302. [CrossRef]

9. Isham, C.J.; Salam, A.; Strathdee, J. f-Dominance of Gravity. Phys. Rev. 1971, D3, 867-873. [CrossRef]

10. Damour, T.; Kogan, I. Effective Lagrangians and universality classes of nonlinear bigravity. Phys. Rev. 2002, D66, 104024. [CrossRef]

11. Capozziello, S.; Faraoni, V. Beyond Einstein Gravity: A Survey of Gravitational Theories for Cosmology and Astrophysics; Springer: New York, NY, USA, 2011.

12. Bajardi, F.; Bascone, F.; Capozziello, S. Renormalizability of Alternative Theories of Gravity: Differences between Power Counting and Entropy Argument. Universe 2021, 7, 148. [CrossRef]

13. Vernov, S.; Pozdeeva, E. De Sitter Solutions in Einstein-Gauss-Bonnet Gravity. Universe 2021, 7, 149. [CrossRef]

14. Pfeifer, C.; Schuster, S. Static Spherically Symmetric Black Holes in Weak f(T)-Gravity. Universe 2021, 7, 153. [CrossRef]

15. Hassan, S.F.; Rosen, R.A. Bimetric Gravity from Ghost-free Massive Gravity. J. High Energy Phys. 2012, 1202, 126. [CrossRef]

16. Hassan, S.F.; Rosen, R.A. Confirmation of the Secondary Constraint and Absence of Ghost in Massive Gravity and Bimetric Gravity. J. High Energy Phys. 2012, 1204, 123. [CrossRef]

17. Hassan, S.F.; Rosen, R.A. Resolving the Ghost Problem in non-Linear Massive Gravity. Phys. Rev. Lett. 2012, $108,041101$. [CrossRef]

18. Hassan, S.F.; Rosen, R.A.; Schmidt-May, A. Ghost-free Massive Gravity with a General Reference Metric. J. High Energy Phys. 2012, 1202, 026. [CrossRef]

19. Soloviev, V.O.; Tchichikina, M.V. Bigravity in Kuchař's Hamiltonian formalism. 1. The general case. Theor. Math. Phys. 2013, 176, 1163-1175. [CrossRef]

20. Soloviev, V.O.; Tchichikina, M.V. Bigravity in Kuchar̆'s Hamiltonian formalism. 2. The special case. Phys. Rev. 2013, D88, 084026. [CrossRef]

21. Hassan, S.F.; Lundkvist, A. Analysis of Constraints and their Algebra in Bimetric Theory. J. High Energy Phys. 2018, 08 , 182. [CrossRef]

22. Hinterbichler, K.; Rosen, R.A. Interacting Spin-2 Fields. J. High Energy Phys. 2012, 7, 47. [CrossRef]

23. Alexandrov, S.; Krasnov, K.; Speziale, S. Chiral description of ghost-free massive gravity. J. High Energy Phys. 2013, 6, 68. [CrossRef]

24. Alexandrov, S. Canonical structure of Tetrad Bimetric Gravity. Gen. Rel. Grav. 2014, 46, 1639. [CrossRef]

25. Soloviev, V.O. Bigravity in tetrad Hamiltonian formalism and matter couplings. Theor. Math. Phys. 2015, 182, 294-307. [CrossRef]

26. Soloviev, V.O. Constraint algebra in tetrad bigravity. Class. Quantum Gravity 2020, 38, 025007. [CrossRef]

27. Kocic, M. Geometric Mean of Bimetric Spacetimes. Class. Quantum Gravity 2021, 38, 075023. [CrossRef]

28. Dirac, P.A.M. Generalized Hamiltonian Dynamics. Can. J. Math. 1950, 2, 129-148. [CrossRef]

29. Dirac, P.A.M. Lectures on Quantum Mechanics; Belfer Graduate School of Science, Yeshiva University: New York, NY, USA, 1964. 
30. Fairlie, D.; Leznov, A. General solutions of the Monge-Ampère equation in n-dimensional space. J. Geom. Phys. 1995, 16, 385-390. [CrossRef]

31. Chaundy, T. The Differential Calculus; Clarendon Press: Oxford, UK, 1935.

32. Hassan, S.F.; Kocic, M. On the local structure of spacetime in ghost-free bimetric theory and massive gravity. J. High Energ. Phys. 2018, 2018, 99. [CrossRef]

33. Kuchar̆, K. Canonical Quantization of Gravity. In Relativity, Astrophysics and Cosmology, Proceedings of the Summer School Held, at the Banff Centre, Banff, AB, Canada, 14-26 August 1972; Israel, W., Reidel, D., Eds.; Springer: Dordreht, The Netherlands; Boston, MA, USA, 1973.

34. Kuchar̆, K. Geometry of hyperspace. I. J. Math. Phys. 1976, 17, 777-791. [CrossRef]

35. Kuchar̆, K. Kinematics of tensor fields in hyperspace. II. J. Math. Phys. 1976, 17, 792-800. [CrossRef]

36. Kuchar̆, K. Dynamics of tensor fields in hyperspace. III. J. Math. Phys. 1976, 17, 801-820. [CrossRef]

37. Kuchar̆, K. Geometrodynamics with tensor sources. IV. J. Math. Phys. 1977, 18, 1589-1597. [CrossRef]

38. York, J.W., Jr. Kinematics and Dynamics of General Relativity. In Sources of the Gravitational Radiation, Proceedings of the Battelle Seattle Workshop, Seattle, WA, USA, 24 July-4 August 1978; Smarr, L.L., Ed.; Center for Astrophysics and Lyman Laboratory of Physics Harvard University: Harvard, MA, USA, 1978.

39. Arnowitt, R.; Deser, S.; Misner, C.W. The Dynamics of General Relativity. In Gravitation, an Introduction to Current Research; Witten, L., Ed.; Wiley: New York, NY, USA, 1963.

40. Comelli, D.; Crisostomi, M.; Nesti, F.; Pilo, L. Degrees of freedom in massive gravity. Phys. Rev. 2012, D86, 101502(R). [CrossRef]

41. Comelli, D.; Nesti, F.; Pilo, L. Weak Massive Gravity. Phys. Rev. 2013, D87, 124021. [CrossRef]

42. Comelli, D.; Nesti, F.; Pilo, L. Massive gravity: A General Analysis. J. High Energy Phys. 2013, 07, 161. [CrossRef]

43. Teitelboim, C. How commutators of constraints reflect the spacetime structure. Ann. Phys. 1973, 79, 542-557. [CrossRef]

44. Hojman, S.; Kuchar̆, K.; Teitelboim, C. Geometrodynamics Regained. Ann. Phys. 1976, 96, 88-135. [CrossRef]

45. Deser, S.; Isham, C.J. Canonical vierbein form of general relativity. Phys. Rev. 1976, D14, 2505-2510. [CrossRef]

46. Nelson, J.E.; Teitelboim, C. Hamiltonian formulation of the theory of interacting gravitational and electron fields. Ann. Phys. 1978, 116, 86-104.

47. Henneaux, M. On geometrodynamics with tetrad fields. Gen. Rel. Grav. 1978, 9, 1031-1045. [CrossRef]

48. Henneaux, M. Poisson brackets of the constraints in the Hamiltonian formulation of tetrad gravity. Phys. Rev. 1983, D27, 986-989. [CrossRef]

49. Kluson, J. Hamiltonian Formalism of Bimetric Gravity In Vierbein Formulation. Eur. Phys. J. 2014, C74, 2985. [CrossRef]

50. Hassan, S.F.; Kocic, M.; Schmidt-May, A. Absence of ghost in a new bimetric-matter coupling. arXiv 2014, arXiv:1409.1909.

51. Kocic, M. Causal Propagation of Constraints in Bimetric Relativity in Standard 3+1 Form. J. High Energy Phys. 2019, 10, 219. [CrossRef] 